\title{
Coloración de aceros inoxidables AISI 304 y AISI 316L para uso biomédico
}

\section{Coloration of the AISI 304 and AISI 316L stainless steels for biomedical applications}

\author{
Luis Fernando Padilla Jiménez \\ Ingeniero Metalúrgico, \\ Universidad Industrial de Santander,Colombia. \\ aguilucho129@hotmail.com \\ Jáder Enrique Guerrero Bermúdez \\ Ph. D. Ciencias Naturales-Física, \\ Universidad Industrial de Santander, Colombia. \\ jader@uis.edu.co
}

\author{
Nayla Julieth Parada Gamboa \\ Ingeniera Química, \\ Universidad Industrial de Santander, Colombia. \\ nayla_parada@hotmail.com \\ Fernando Viejo Abrante \\ Ph. D. Ciencia y Tecnología de Materiales, GIMAT, \\ Universidad Industrial de Santander, Colombia. \\ fviejo@uis.edu.co
}

\begin{abstract}
Ana Emilse Coy Echeverría
Ph. D. Ciencia y Tecnología de Materiales, GIMAT,

Universidad Industrial de Santander, Colombia.

anaecoy@uis.edu.co
\end{abstract}

\begin{abstract}
Resumen-En el presente trabajo se desarrollaron películas de óxido de cromo, sobre la superficie de los aceros inoxidables AISI 304 y $316 \mathrm{~L}$ mediante tratamientos químicos y de endurecimiento electroquímico que puedan presentar aplicación en los bioimplantes. De esta manera, se evaluó la influencia de distintas variables en ambos tratamientos; tales como tiempo sobre la coloración (tratamiento químico), y la densidad de corriente aplicada para un tiempo constante (endurecimiento electroquímico). La caracterización de las películas de cromo obtenidas se llevó a cabo mediante técnicas de espectrometría, reflectancia y microscopía óptica. De forma complementaria, se evaluó la estabilidad electroquímica de las películas formadas mediante ensayos de polarización anódica en solución de Hank a $37^{\circ} \mathrm{C}$. Los resultados demostraron que, bajo determinadas condiciones experimentales, es posible obtener películas homogéneas y reproducibles con una amplia gama de coloraciones. Por otra parte, los resultados electroquímicos mostraron que las películas obtenidas desarrollaron un cambio en el mecanismo de ataque de ambos aceros.
\end{abstract}

Palabras clave- Aceros inoxidables austeníticos, Biomateriales, Coloración.

Abstract- In this work, chromium oxide films were developed on the surface of the AISI 304 and 316L stainless steels by chemical and electrochemical hardening treatments. Thus, the influence of different variables in both treatments (i.e. immersion time, current density applied,...) was investigated. Characterization of the chromium films was carried out using spectrometry, reflectance and optical microscopy techniques. Further, electrochemical stability of the films was evaluated by potentiodynamic anodic polarization in Hank's solution at $37^{\circ} \mathrm{C}$. The results showed that, under some specific conditions, it was possible to obtain homogeneous and reproducible chromium oxide films with a wide range of colors. On the other hand, the electrochemical results showed that the films formed modified the corrosion mechanism of both stainless steels.

Keywords- Austenitic stainless steels, Biomaterials, Coloration.

\section{INTRODUCCIÓN}

Los biomateriales son sustancias sintéticas o naturales utilizadas para sustituir partes del organismo humano o para funcionar en contacto íntimo con tejidos vivos. Los biomateriales metálicos han sido utilizados en implantes biomédicos ortopédicos y odontológicos, llegando a ser indispensables en aquellos casos en que las solicitaciones mecánicas a las que éstos están sometidos durante el servicio son extremas [1].

Las excelentes propiedades mecánicas y químicas de las aleaciones de Ti y las aleaciones de $\mathrm{Co}-\mathrm{Cr}$ han hecho de ellos los biomateriales metálicos más utilizados en implantes ortopédicos [2],[3]. Sin embargo, su gran desventaja es el alto coste de producción.

La necesidad de reducción de costes ha llevado al uso extendido de otros materiales en la cirugía ortopédica como son los aceros inoxidables 
austeníticos. Estas aleaciones, además de poseer un reducido coste comparado con las aleaciones de $\mathrm{Ti}$, tienen buenas propiedades mecánicas y son relativamente fáciles de producir. Además, los aceros inoxidables presentan la característica especial de poseer una delgada película inerte de óxido de cromo $\left(\mathrm{Cr}_{2} \mathrm{O}_{3}\right)$ sobre su superficie, que protege el metal frente a la corrosión en la mayoría de los ambientes de trabajo. Sin embargo, en ocasiones, contactos prolongados con los fluidos fisiológicos del cuerpo humano pueden dar lugar a fenómenos de corrosión y al debilitamiento del implante. La liberación de iones de Ni (II) durante el proceso de corrosión, puede causar enfermedades citotóxicas como inflamación, alergia, necrosis, tesaurismosis o cáncer [4].

Una posible solución a este problema consiste en favorecer el crecimiento de la película de óxido de cromo superficial mediante tratamientos químicos o electroquímicos, con el fin de proteger el implante de acero y, de esta forma, evitar la liberación de iones metálicos que puedan causar daños al organismo del paciente. Ambos tipos de tratamiento permiten obtener películas de óxido y realizar un control adecuado de espesores de película del recubrimiento mediante la regulación de variables tales como la temperatura de la solución, la densidad de corriente, el tipo y/o la diferencia de potencial [5]-[9].

De manera adicional, una característica muy particular de estos tratamientos radica en que durante el crecimiento de la película de óxido de cromo sobre la superficie del acero inoxidable se modifica su coloración, permitiendo obtener una gama amplia de colores que van desde el dorado hasta el azul o verde. Dicha característica ha encontrado una aplicación muy interesante, ya que en la actualidad muchas empresas dedicadas al diseño y producción de bioimplantes utilizan la coloración del acero como parámetro principal para poder identificar y clasificar de forma rutinaria la gran variedad de componentes que se fabrican para aplicaciones diversas (tornillos, clavos, etc.). Recientemente sehan estudiado otras tecnologías de coloración de aceros inoxidables como lo es el uso de radiación láser; sin embargo, esta tecnología presenta problemas de reproducibilidad en el color y estabilidad de proceso, de forma que aún no ha sido posible su aplicación industrial [10][12].
El presente trabajo tiene como objetivo desarrollar películas de óxido de cromo sobre la superficie de los aceros inoxidables AISI 304 y 316L, mediante tratamientos químicos y electroquímicos, que puedan presentar aplicación en la fabricación de bioimplantes. En este sentido, se evaluará la influencia de las distintas variables de ambos tratamientos, con el fin de obtener películas de coloración homogénea y mejorar la reproducibilidad del color. Además, de forma complementaria se evaluará la estabilidad química (resistencia a la corrosión) de las películas obtenidas en un medio fisiológico artificial.

\section{PROCEDIMIENTO EXPERIMENTAL}

\subsection{Materiales y preparación}

Los materiales utilizados en el desarrollo de este trabajo fueron los aceros inoxidable AISI 304 y AISI 316L suministrados por la empresa IMPORINOX S.A. La composición química (\% en masa) de los aceros fue: i) AISI 304: 0,07C, 2,0Mn, 1,0Si, $18 \mathrm{Cr}, 9 \mathrm{Ni}, 0,045 \mathrm{P}, 0,015 \mathrm{~S}$ y $\mathrm{Fe}$ como balance; ii) AISI 316L: 0,03C, 2,0Mn, 1,0Si, $18 \mathrm{Cr}, 12 \mathrm{Ni}, 2,0 \mathrm{Mo}$ 0,045P, 0,015S y Fe como balance.

La preparación superficial de las muestras a colorear se realizó mediante desbaste en medio acuoso con papel abrasivo de $\mathrm{SiC}$ hasta granulometría 600. Adicionalmente, las muestras fueron sometidas a una limpieza posterior en baño de ultrasonidos en una solución de ácido nítrico al $9 \%$ a $60{ }^{\circ} \mathrm{C}$ durante 15 minutos para remover posibles impurezas que no fueron eliminadas en la etapa anterior. Para terminar, el proceso de limpieza, las muestras se lavaron con agua destilada.

\subsection{Tratamiento de coloración química}

La coloración mediante tratamiento químico se llevó a cabo mediante la inmersión de las muestras de acero en una disolución compuesta de 5,0 M ácido sulfúrico y 2,5 M ácido crómico, en distintos tiempos comprendidos entre 15 y 80 minutos, a una temperatura de $80{ }^{\circ} \mathrm{C}$ y con agitación constante. Una vez finalizado el tratamiento de coloración las muestras se extrajeron de la disolución, se lavaron con agua destilada y se secaron. 
2.3 Tratamiento de endurecimiento electroquímico

El tratamiento de endurecimiento electroquímico se llevó a cabo con el fin de mejorar la tonalidad, la uniformidad y las propiedades de la película de óxido de cromo formada sobre la superficie de la probeta, mediante la imposición de una corriente externa.

De este modo, el proceso se dividió en dos etapas: una primera etapa previa en la cual se les hizo un tratamiento químico a las muestras de acero, como se describió en el apartado anterior. En la segunda etapa, tratamiento de endurecimiento electroquímico, las muestras fueron tratadas por 10 minutos en una celda electroquímica con una solución 0,026 M de ácido sulfúrico y 2,5 $\mathrm{M}$ de ácido crómico. La muestra se conectó como ánodo y como cátodo se utilizó un electrodo inerte de platino. Se aplicaron densidades de corriente que variaron entre 5,0 y $6,0 \mathrm{~mA} / \mathrm{cm}^{2}$.

\subsection{Evaluación cromática de los aceros coloreados y cálculo de espesores de las películas}

Se realizó la evaluación cromática con el fin de evaluar el efecto de las distintas variables de tratamiento en la coloración de cada uno de los recubrimientos obtenidos (tiempo, densidad de corriente, tipo de acero). Para tal fin se utilizó un espectrofotómetro USB 4000 de Ocean Optics acoplado a una esfera integradora que exhibe la geometría $8 \%$ d. Cada color resultante se determinó mediante métodos gráficos y computacionales (Mathlab). El resultado fue la obtención de las coordenadas cromáticas $\mathrm{x}$ e $\mathrm{y}$, que ubican el color dentro del diagrama de cromaticidad; la respectiva longitud de onda dominante o característica, captada mediante el espectrofotómetro usado, y que permite conocer la ubicación del color en el espectro visible; y el grado de luminosidad (espacio SCIELAB). A partir de la obtención de estos parámetros cromáticos se pudo también calcular las longitudes de onda de reflectancia mínima ( $\lambda \min )$ y máxima ( $\lambda$ máx) que permitieron determinar un valor estimado del espesor de la capa de óxido formada en función de las variables estudiadas para ambos tipos de tratamiento, de acuerdo con la siguiente ecuación, válida para el análisis de capas dieléctricas, tales como el óxido de cromo:

$$
D=\frac{\lambda \min }{4 n\left(1-\frac{\lambda \min }{\lambda \max }\right)}(1)
$$

$D$ : es el espesor de la capa de óxido.

$n$ : es el índice de refracción del óxido de cromo asumido como 2,2.

$\lambda$ min: es el valor de la longitud de onda donde ocurre la mínima reflexión de la luz de la probeta tratada en la esfera integradora.

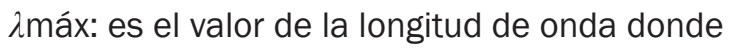
ocurre la máxima reflexión de la luz de la probeta tratada en la esfera integradora.

\subsection{Evaluación de la estabilidad electroquímica}

Se realizaron ensayos de polarización potenciodinámica con el fin de evaluar la resistencia a la corrosión por picadura de los aceros coloreados frente a la acción agresiva de un medio fisiológico artificial (solución de Hank) a pH 7,0 y a una temperatura de $37^{\circ} \mathrm{C}$.

El ensayo se realizó mediante un potenciostato marca Gill AC de ACMInstruments. Las pruebas se efectuaron en una celda electroquímica de tres electrodos con electrodo auxiliar de platino, un electrodo de referencia de calomelanos y como electrodo de trabajo la muestra evaluada.

Previo al ensayo de polarización potenciodinámica, las muestras se dejaron estabilizar en la solución durante $1 \mathrm{~h}$. Una vez estabilizado el sistema se determinó el potencial de circuito abierto (OCP) y se varió el potencial aplicado desde -100 $\mathrm{mV}$ hasta $+2000 \mathrm{mV}$ respecto al valor de OCP. La densidad de corriente registrada se limitó en 0,01 $\mathrm{A} / \mathrm{cm}^{2}$.

\section{RESULTADOS Y DISCUSIÓN}

\subsection{Evaluación cromática de los aceros coloreados y cálculo de espesores de las películas}

\subsubsection{Coloración química}

La Fig. 1 muestra el aspecto superficial de las probetas del acero inoxidable AISI 304 coloreado químicamente a $80{ }^{\circ} \mathrm{C}$, en función del tiempo de inmersión en la solución de tratamiento (de 40 
a 60 minutos). Se puede observar que, para las condiciones estudiadas, la coloración superficial obtenida fue uniforme y varió en función del tiempo de inmersión, obteniéndose coloraciones, para el acero AISI 304, desde un color marrón oscuro a 40 minutos hasta un color verde para 60 minutos de tratamiento, y para el acero AISI 316L desde un café grisáceo para un tiempo de 40 minutos, desde azules y dorados, hasta el verde dorado para un tiempo de tratamiento de 60 minutos. Cabe mencionar que, dichos colores fueron reproducibles para cada uno de los tiempos de inmersión.

Con relación a la caracterización cromática, las Figs. 2 y 3 muestran los diagramas de cromaticidad para el acero AISI 304 sin tratamiento y para las probetas tratadas químicamente a diferentes tiempos de inmersión, respectivamente.

A partir de estos diagramas se determinaron las longitudes de onda dominantes bajo la radiación del iluminante A. Para el caso específico del acero AISI 304, sin tratamiento, este valor se estimó en 583nm. Los valores determinados en el diagrama de cromaticidad de la Fig. 3 se recogen en la Tabla I, al igual que los valores de los parámetros cromáticos determinados en función del tiempo de inmersión, tanto para el acero AISI 304 como para el AISI 316 L coloreados químicamente. Al comparar los valores de la longitud de onda dominante obtenida directamente del espectrofotómetro con aquéllos obtenidos mediante el diagrama de cromaticidad, se observó que el error en la determinación fue mínimo, corroborándose los valores reales de las longitudes de onda dominantes características de los colores obtenidos.

Por otro lado, el grado de luminosidad de cada color no muestra tendencias de crecimiento o disminución en función del tiempo de inmersión, al igual que las longitudes de onda dominante de los colores resultantes. En este sentido, por ejemplo, el valor de la longitud de onda dominante del coIor producido en el proceso, sobre el acero AISI 304, a 55 minutos (azul) es diferente a los demás valores. Esto es debido a que el color azul, por su naturaleza cromática y su gama de tonalidades, se ubica en el espectro visible con longitudes de onda que oscilan entre 430 y $480 \mathrm{~nm}$. Por consiguiente, no se puede relacionar el crecimiento de la capa de óxido en función de estos dos factores de manera directa, ni relacionar los colores obte- nidos con efectos de formación de la capa de óxido de cromo. Dicha relación, no obstante, puede determinarse mediante el análisis de los espectros de reflectancia y el cálculo de la longitud de onda a las cuales ocurre la reflectancia mínima ( $\lambda$ min) y máxima ( $\lambda$ máx) para cada color obtenido a partir de los tratamientos de coloración.

Fig. 1. ACEROS INOXIDABLES AISI 304 Y AISI 316 L SIN COLOREAR Y COLOREADOS QUÍMICAMENTE A $80^{\circ} \mathrm{C} Y$ DISTINTOS TIEMPOS DE TRATAMIENTO.

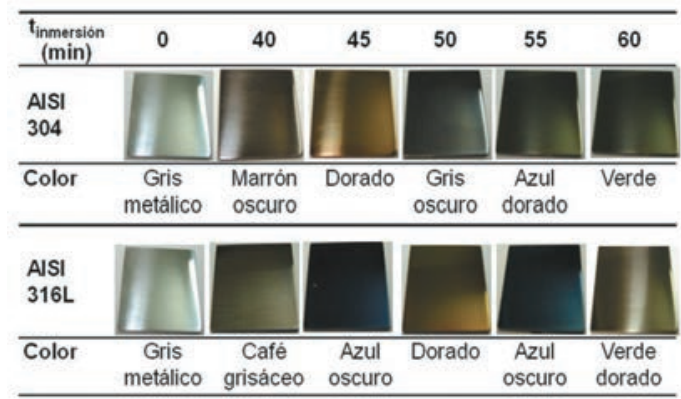

Fig. 2. DIAGRAMA DE CROMATICIDAD PARA EL ACERO AISI 304 SIN RECUBRIMIENTO

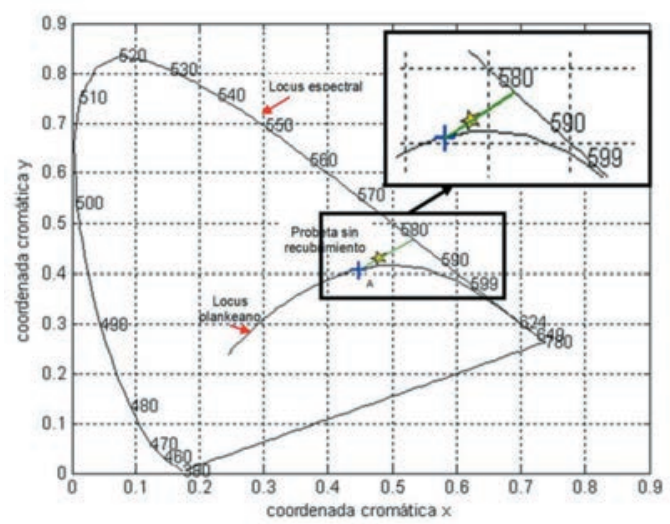

Fig. 3. DIAGRAMA DE CROMATICIDAD PARA EL ACERO AISI 304 COLOREADO QUIIMICAMENTE A DISTINTOS TIEMPOS DE INMERSIÓN.

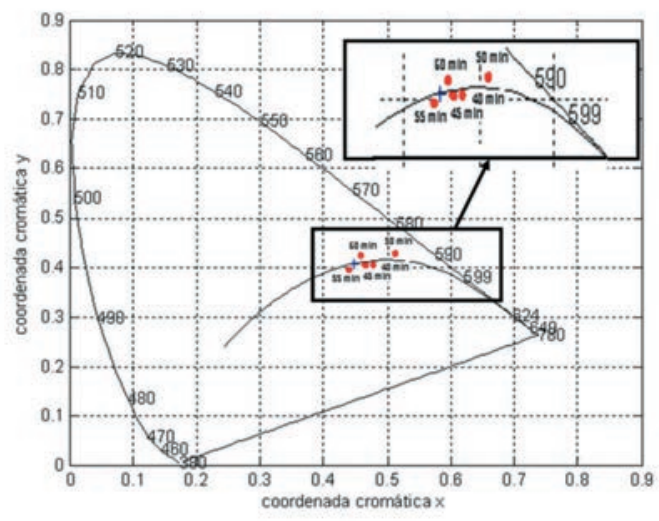


TABLA I

PARÁMETROS DE CARACTERIZACIÓN CROMÁTICA PARA LOS ACEROS AISI 304 Y AISI 316L COLOREADOS QUÍMICAMENTE A DISTINTOS TIEMPOS

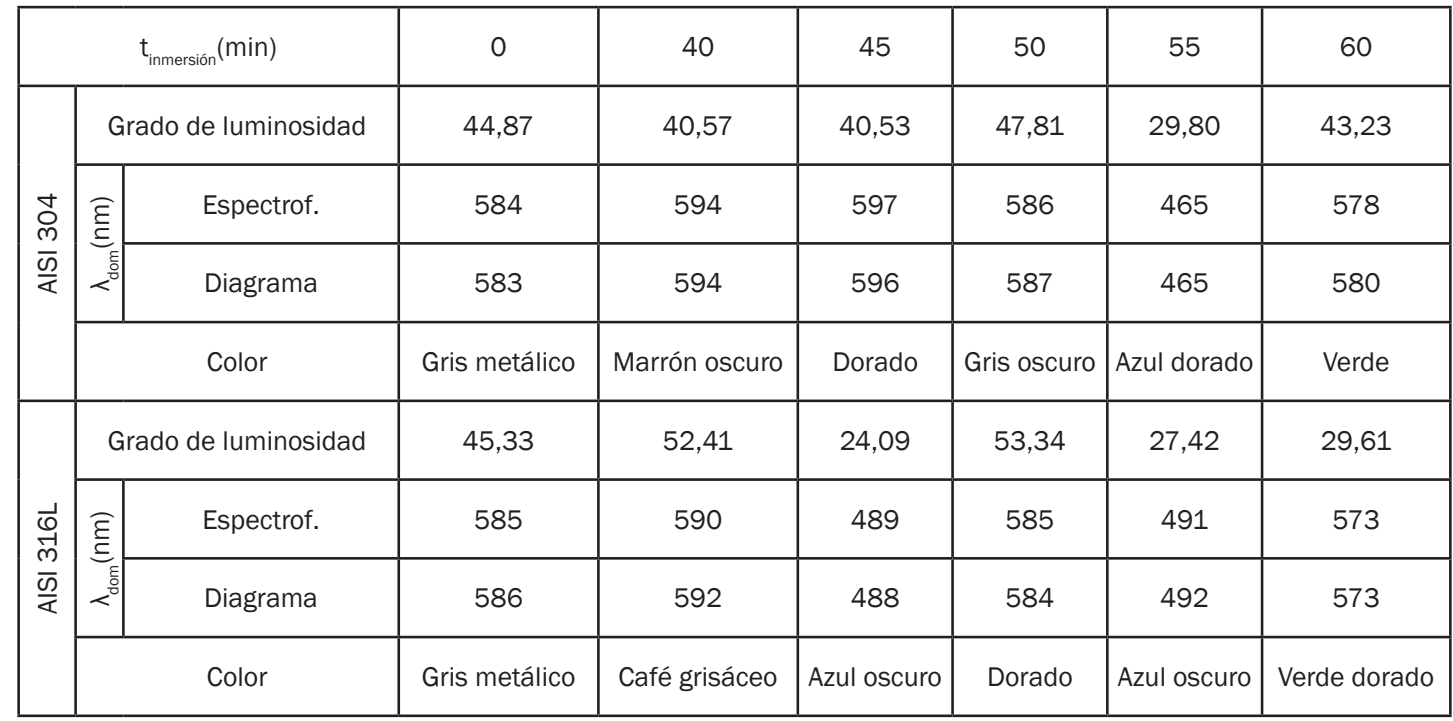

TABLA II

ESPESORES DE LAS CAPAS DE ÓXIDO DE CROMO DE LOS ACEROS AISI 304 Y AISI 316L COLOREADOS QUÍMICAMENTE A DISTINTOS TIEMPOS

\begin{tabular}{|c|c|c|c|c|c|c|}
\hline \multicolumn{2}{|c|}{$\mathrm{t}_{\text {inmersión }}(\mathrm{min})$} & 40 & 45 & 50 & 55 & 60 \\
\hline \multirow{3}{*}{$\begin{array}{l}\searrow \\
\text { ○ } \\
\frac{\bar{N}}{\pi}\end{array}$} & $\lambda_{\min }(n m)$ & 440 & 440 & $435 \mid$ & 437 & 600 \\
\hline & $\lambda_{\text {máx }}(n m)$ & 775 & 775 & 650 & 555 & 775 \\
\hline & Espesor (nm) & 116 & 116 & 149 & 233 & 301 \\
\hline \multirow{3}{*}{$\begin{array}{l}\vec{\sigma} \\
\frac{\vec{m}}{\bar{m}} \\
\frac{\bar{\sigma}}{\pi}\end{array}$} & $\lambda_{\min }(n m)$ & 437 & 700 & 675 & 460 & 575 \\
\hline & $\lambda_{\text {máx }}(n m)$ & 775 & 430 & 430 & 590 & 775 \\
\hline & Espesor (nm) & 114 & 126 & 134 & 237 & 253 \\
\hline
\end{tabular}

En este sentido, en la Tabla II se listan los valores correspondientes de $\lambda$ min y $\lambda$ máx, así como el espesor de la capa de óxido de cromo, calculado mediante la ecuación 1, para los dos aceros, en función del tiempo de tratamiento de coloración. En general, se observa que la película crece en función del tiempo de tratamiento para ambos aceros.

Finalmente, en la Fig. 4 se representa el espesor estimado de película en función del tiempo de tratamiento. En general, la gráfica muestra cómo el espesor de la película de óxido crece en función del tiempo de tratamiento para ambos aceros. Sin embargo, para tiempos prolongados (por encima de 55 minutos) se observó un comportamiento diferente. En este sentido, se podrían identificar tres etapas de crecimiento de la película de óxido de cromo dentro del proceso de coloración química.
Fig. 4. CRECIMIENTO DE LA CAPA DE ÓXIDO DE CROMO EN FUNCIÓN DEL TIEMPO DE TRATAMIENTO DE COLORACIÓN QUÍMICA PARA LOS ACEROS INOXIDABLES ESTUDIADOS.

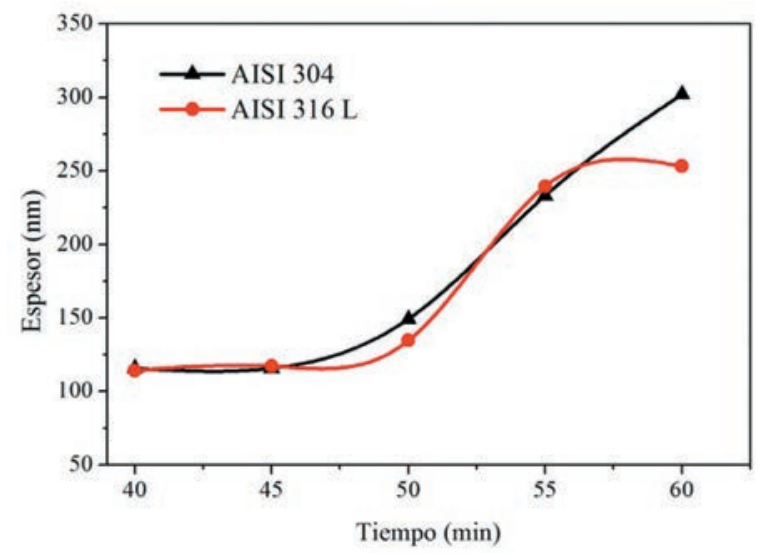

La primera de ellas ocurriría entre los 40 y 50 minutos aproximadamente, y sería una etapa de 
incubación donde se empezaría a generar la película de óxido de cromo. La segunda etapa transcurriría a partir de los 50 minutos de tratamiento, en la cual se observa un aumento lineal del espesor con el tiempo de tratamiento que podría asociarse al crecimiento lineal de la película. Este comportamiento, en el caso del acero AISI 304, continúa observándose para los mayores tiempos de tratamiento (60 minutos). Sin embargo, para el caso del acero AISI 316L, por encima de los 55 minutos de tratamiento de coloración, su espesor no se ve incrementado, hecho que se podría asociar a una tercera etapa o periodo de estabilización de la película de óxido: es bien conocido que el molibdeno como elemento aleante del acero AISI 316L, incrementa la estabilización de las películas de óxido de cromo y dificulta su crecimiento de forma continua [13].

\subsubsection{Coloración química más endurecimiento electroquímico}

En la Fig. 5 se muestran las imágenes del acero AISI 316L después del tratamiento de coloración química a $80^{\circ} \mathrm{C}$ por tiempos de 40 a 60 minutos y posterior endurecimiento electroquímico, aplicando densidades de corriente de 5,0 a $6,0 \mathrm{~mA} / \mathrm{cm}^{2}$. Las imágenes confirman que los tratamientos de coloración química con endurecimiento electroquímico permiten también obtener una gran variedad de colores, siendo éstos homogéneos y reproducibles para cada una de las condiciones estudiadas.

La caracterización cromática para los dos aceros estudiados, llevada a cabo a partir de los diagramas de cromaticidad se resume en la Tabla III. Como se encontró en los resultados de coloración química, los valores determinados de longitud de onda dominante obtenidos mediante el diagrama de cromaticidad y mediante el espectrofotómetro fueron muy similares. Así mismo, el valor de dicha longitud de onda dominante y del grado de luminosidad, no mostraron ninguna tendencia de aumento o disminución en función del tiempo de coloración o de la corriente de endurecimiento.

En el caso del acero AISI 304, a medida que se aumenta tanto el tiempo de coloración química como la densidad de corriente aplicada durante la etapa de endurecimiento electroquímico, se pre- sentan pequeñas variaciones en la coloración de las muestras. Esto, de manera general, da como resultado un cambio de color desde gris metálico hacia una gama de colores resultado de la combinación de los colores azul, verde y dorado.

En el caso del acero AISI 316L, para un tiempo de 40 min de coloración química, la variación de la densidad de corriente modifica muy poco las coloraciones obtenidas, que se encuentran cercanas al color gris original. Este fenómeno podría asociarse a un crecimiento mínimo de las capas de óxido de cromo bajo estas condiciones de tratamiento; mientras que, para mayores tiempos de inmersión durante el tratamiento químico (50 y $60 \mathrm{~min}$ ) y aumentando la densidad de corriente se altera las coordenadas cromáticas. Como resultado se obtienen nuevos colores (rojos, dorados y azules) totalmente distintos al gris original y su gama de tonalidades. Es importante resaltar que, en general, se obtuvo mayor variación de coloraciones en el acero AISI 316L comparada con acero el acero AISI 304.

Por otro lado, en las Figs. 6 y 7 se representa el espesor de la película de óxido de cromo formada en función del tiempo y la densidad de corriente aplicada, sobre los aceros inoxidables AISI 304 y AISI 316L, respectivamente.

Fig. 5. ACERO INOXIDABLE AISI 316L DESPUÉS DE LA COLORACIÓN QUÍMICA A $80^{\circ} \mathrm{C} Y$ A DIFERENTES TIEMPOS, MÁS POSTERIOR ENDURECIMIENTO ELECTROQUÍMICO A DIFERENTES DENSIDADES DE CORRIENTE

\begin{tabular}{|c|c|c|c|}
\hline $\mathrm{i}\left(\mathrm{mA} / \mathrm{cm}^{2}\right)$ & & & \\
\hline $\begin{array}{l}t_{\text {inmersión }} \\
(\mathrm{min})\end{array}$ & 5,0 & 5,5 & 6,0 \\
\hline 40 & & & \\
\hline Color & Gris claro & $\begin{array}{c}\text { Gris } \\
\text { dorado }\end{array}$ & Gris oscuro \\
\hline 50 & & & \\
\hline Color & Azul oscuro & $\begin{array}{l}\text { Azul } \\
\text { mate }\end{array}$ & Rojo \\
\hline 60 & & & \\
\hline Color & $\begin{array}{l}\text { Dorado } \\
\text { verdoso }\end{array}$ & $\begin{array}{c}\text { Azul } \\
\text { oscuro }\end{array}$ & $\begin{array}{c}\text { Dorado } \\
\text { Rojizo }\end{array}$ \\
\hline
\end{tabular}


TABLA III

PARÁMETROS DE CARACTERIZACIÓN CROMÁTICA PARA LOS ACEROS AISI 304 Y AISI 316L COLOREADOS QUÍMICAMENTE A 80C Y DIFERENTES TIEMPOS DE INMERSIÓN MÁS POSTERIOR ENDURECIMIENTO ELECTROQUÍMICO A DIFERENTES DENSIDADES DE CORRIENTE

\begin{tabular}{|c|c|c|c|c|c|c|}
\hline \multirow{2}{*}{$\begin{array}{l}\text { 잉 } \\
\text { 운 }\end{array}$} & \multirow{2}{*}{$\mathrm{t}_{\text {inmersión }}(\mathrm{min})$} & \multirow{2}{*}{$\begin{array}{c}\mathrm{i} \\
\left(\mathrm{mA} / \mathrm{cm}^{2}\right)\end{array}$} & \multirow{2}{*}{ Grado de luminosidad } & \multicolumn{2}{|c|}{$\lambda_{\text {dominante }}(\mathrm{nm})$} & \multirow{2}{*}{ Color percibido por el ojo humano } \\
\hline & & & & Espect.. & Diagram & \\
\hline \multirow{9}{*}{$\begin{array}{l}\text { ঠे } \\
\frac{\text { ले }}{\text { ब }}\end{array}$} & 40 & \multirow{3}{*}{5,0} & 50,76 & 591 & 592 & Gris oscuro \\
\hline & 50 & & 41,50 & 558 & 560 & Verde dorado \\
\hline & 60 & & 31,61 & 505 & 507 & Azul oscuro \\
\hline & 40 & \multirow{3}{*}{5,5} & 43,05 & 569 & 572 & Verde dorado \\
\hline & 50 & & 32,77 & 490 & 488 & Azul oscuro \\
\hline & 60 & & 40,29 & 574 & 576 & Verde \\
\hline & 40 & \multirow{3}{*}{6,0} & 32,00 & 485 & 484 & Azul - verdoso \\
\hline & 50 & & 31,87 & 495 & 495 & Verde dorado \\
\hline & 60 & & 40,72 & 578 & 579 & Dorado verdoso \\
\hline \multirow{9}{*}{$\begin{array}{l}\overrightarrow{0} \\
\frac{\vec{m}}{\bar{\omega}} \\
\frac{\bar{\alpha}}{\alpha}\end{array}$} & 40 & \multirow{3}{*}{5,0} & 59,58 & 589 & 591 & Gris claro \\
\hline & 50 & & 46,48 & 498 & 497 & Azul oscuro \\
\hline & 60 & & 51,19 & 582 & 582 & Dorado verdoso \\
\hline & 40 & \multirow{3}{*}{5,5} & 59,29 & 590 & 589 & Gris dorado \\
\hline & 50 & & 32,47 & 491 & 490 & Azul mate \\
\hline & 60 & & 25,10 & 489 & 488 & Azul oscuro \\
\hline & 40 & \multirow{3}{*}{6,0} & 59,30 & 593 & 590 & Gris oscuro \\
\hline & 50 & & 44,75 & 605 & 607 & Rojo \\
\hline & 60 & & 50,38 & 588 & 590 & Dorado rojizo \\
\hline
\end{tabular}

Fig. 6. ESPESOR DE LA CAPA DE ÓXIDO DE CROMO EN FUNCIÓN DEL TIEMPO DE TRATAMIENTO DE COLORACIÓN QUÍMICA Y DE LA DENSIDAD DE CORRIENTE APLICADA DURANTE EL ENDURECIMIENTO ELECTROQUÍMICO PARA EL ACERO INOXIDABLE AISI 304.

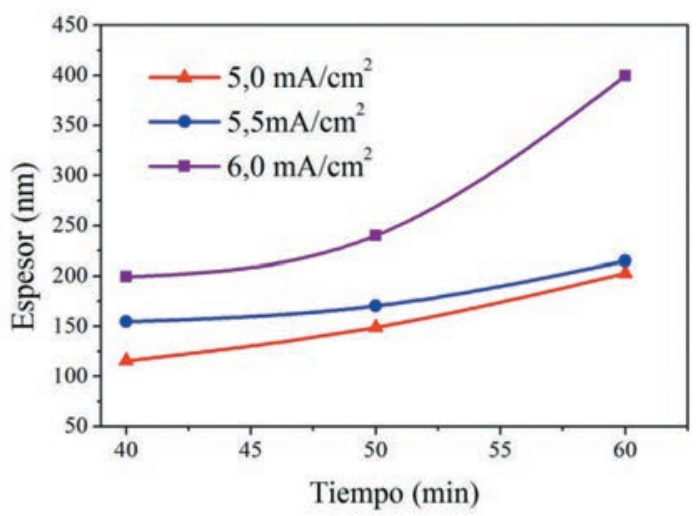

En el caso del acero AISI 304, en general, se observa cómo, al aumentar el tiempo de coloración química y la densidad de corriente aplicada durante la etapa de endurecimiento electroquímico, el espesor de la película de óxido de cromo se ve incrementado de forma notoria, la densidad de corriente es la variable más influyente en el crecimiento de dicha película. También se puede observar que, la cinética de crecimiento de la película es similar para las corrientes de 5,0 y 5,5
$\mathrm{mA} / \mathrm{cm}^{2}$ y es de tipo lineal. Sin embargo, para una corriente de $6,0 \mathrm{~mA} / \mathrm{cm}^{2}$, además de presentar un incremento general en el espesor de las películas obtenidas, existe un cambio de pendiente para un tiempo aproximado de $50 \mathrm{~min}$. A partir de este tiempo se incrementa la velocidad de crecimiento de la película llegándose a obtener espesores de hasta 400nm.

Fig. 7. ESPESOR DE LA CAPA DE ÓXIDO DE CROMO EN FUNCIÓN DEL TIEMPO DE TRATAMIENTO DE COLORACIÓN QUÍMICA Y DE LA DENSIDAD DE CORRIENTE APLICADA DURANTE EL ENDURECIMIENTO ELECTROQUÍMICO PARA EL ACERO INOXIDABLE AISI 316L.

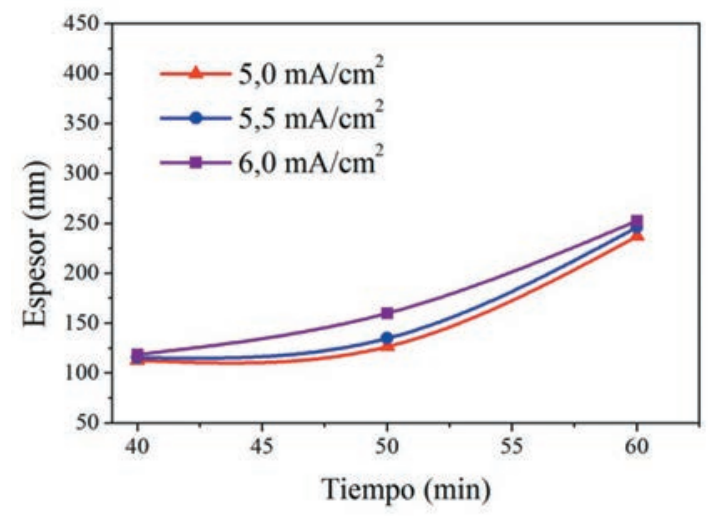

Para el acero AISI 316L (Fig. 7) se observa claramente como el espesor de la película de óxido 
aumenta en función del tiempo de tratamiento de coloración química y de la densidad de corriente aplicada durante el tratamiento de endurecimiento, tal como sucedió en los procesos aplicados al acero AISI 304. Sin embargo, el crecimiento de la película no es muy acusado, obteniéndose valores máximos de 250nm aproximadamente, para el máximo tiempo y la mayor densidad de corriente, (alrededor de un 35\% menor que los alcanzados para el acero AISI 304). Además, se puede apreciar que las cinéticas de crecimiento para todas las densidades de corriente aplicadas son aproximadamente lineales y de menor velocidad con relación a las observadas para el acero AISI 304. Así mismo, el espesor de la película máximo que se obtiene es prácticamente independientemente de la corriente aplicada. Este hecho puede estar asociado, al igual que se observó en los tratamientos de coloración química, a la presencia de molibdeno en la formación de la película de óxido, que estabiliza su estructura y dificulta su crecimiento progresivo.

\subsection{Evaluación de la resistencia a la corrosión}

De forma complementaria a la caracterización cromática, los recubrimientos obtenidos sobre Ios aceros AISI 304 y AISI 316L fueron evaluados mediante ensayos de polarización anódica en un medio fisiológico simulado (solución de Hank) a $37^{\circ} \mathrm{C}$.

\subsubsection{Coloración química}

La Fig. 8 muestra las curvas de polarización anódica del acero inoxidable AISI 304 sin tratamiento y coloreado químicamente durante 40,50 y 60 minutos, después de inmersión en solución de Hank a $37^{\circ} \mathrm{C}$ durante $1 \mathrm{~h}$.

Se puede apreciar cómo la curva de polarización del acero sin tratamiento presenta un típico comportamiento pasivo característico de los aceros inoxidables, presentando una región de pasividad de aproximadamente $290 \mathrm{mV}$. Por otro lado, se puede observar como, para el tratamiento de coloración química durante 40 y 50 minutos, el potencial de corrosión (Ecorr) disminuye obteniéndose valores de -30 y $-230 \mathrm{mV}$, respectivamente. A partir de dicho potencial ambos aceros presentaron también comportamiento pasivo de alrededor de 250 y $450 \mathrm{mV}$.
FIG. 8. CURVA DE POLARIZACIÓN ANÓDICA DEL ACERO INOXIDABLE AISI 304 SIN TRATAMIENTO Y TRATADO MEDIANTE COLORACIÓN QUÍMICA, DESPUÉS DE INMERSIÓN EN SOLUCIÓN DE HANK A $37^{\circ} \mathrm{C}$ DURANTE $1 \mathrm{H}$.

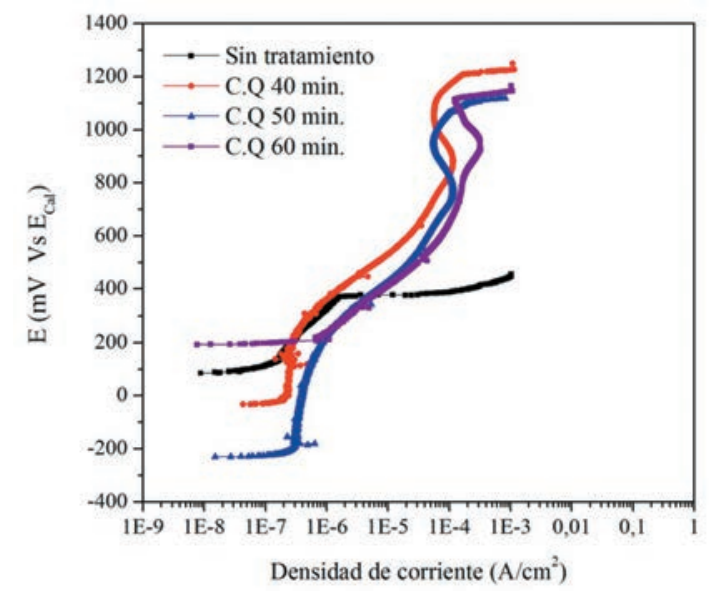

El potencial de picadura $\left(\mathrm{E}_{\mathrm{pic}}\right)$, para las muestras coloreadas, aunque se localiza entre 200 y 350mV, no está bien definido. En esta región, aunque parece existir un proceso de nucleación de picaduras, que podrían ser de carácter metaestable, ya que la ausencia de un cambio acusado en la densidad de corriente indica que el crecimiento de picaduras no se encuentra favorecido. Por encima de esa región, la densidad de corriente se incrementa paulatinamente hasta alcanzar un valor de potencial alrededor de 1100 - 1150mV, donde la película de óxido de cromo finalmente no es estable y se disuelve de forma generalizada [9]. Con relación a la densidad de corriente de corrosión $\left(i_{\text {corr }}\right)$ se determinó para ambos aceros valores de 2,1 y $2,7 \times 10^{-8} \mathrm{~A} / \mathrm{cm}^{2}$ para 40 y 50 minutos respectivamente, que supone un ligero aumento sin llegar a alcanzar el orden de magnitud de diferencia con relación al acero inoxidable sin recubrir.

Por otro lado, el acero tratado durante $60 \mathrm{mi}$ nutos mostró un comportamiento completamente diferente, con un potencial de corrosión mucho más elevado que todas las demás condiciones estudiadas (200mV). Además, la curva de polarización no presentó ningún tipo de comportamiento pasivo que indica que el material se encuentra inicialmente desprotegido frente al proceso de corrosión localizada. Este hecho probablemente estuvo asociado a que, para tiempos de tratamiento de 60 minutos, la superficie ya presentaba un fenómeno de ataque intergranular acusado que dejó al acero ya sensibilizado y desprotegido frente al posterior ensayo de corrosión. En este sentido, la 
TABLA IV

PARÁMETROS CARACTERÍSTICOS DEL ENSAYO DE POLARIZACIÓN ANÓDICA PARA EL ACERO AISI 304 EN DISTINTAS CONDICIONES, SUMERGIDO EN SOLUCIÓN DE HANK A $37^{\circ} \mathrm{C}$ DURANTE 1 HORA

\begin{tabular}{|c|c|c|c|c|c|}
\hline Condición & Ecorr $(\mathrm{mV})$ & Epic $(\mathrm{mV})$ & Rango de Pasividad $(\mathrm{mV})$ & icorr $\left(\mathrm{A} / \mathrm{cm}^{2}\right)$ & ipas $\left(\mathrm{A} / \mathrm{cm}^{2}\right)$ \\
\hline Sin tratamiento & 80 & 370 & 290 & $8,7 \mathrm{E}-09$ & $4,9 \mathrm{E}-07$ \\
\hline 40 minutos & -30 & No def. & 250 aprox. & $2,1 \mathrm{E}-08$ & $2,5 \mathrm{E}-07$ \\
\hline 50 minutos & -230 & No def. & 450 aprox. & $2,7 \mathrm{E}-08$ & $4,5 \mathrm{E}-07$ \\
\hline 60 minutos & 200 & - & - & $1,0 \mathrm{E}-06$ & - \\
\hline
\end{tabular}

densidad de corriente de corrosión se estimó en $1,0 \times 10^{-6} \mathrm{~A} / \mathrm{cm}^{2}$, mayor de un orden de magnitud al valor determinado para el acero sin recubrir. Los parámetros obtenidos de este estudio se resumen en la Tabla IV.

Las micrografías ópticas mostradas en la Fig. 9 confirman dichos resultados. A partir de estas micrografías es evidente que, a pesar de la pasividad mostrada por el acero sin tratar, una vez se alcanza el potencial de picadura, el proceso de nucleación y crecimiento de picaduras se encuentra favorecido, originando picaduras de hasta 200300 micrómetros de diámetro (Fig. 9a). Por otro lado, los aceros tratados por coloración química muestran una superficie uniforme, sin signos evidentes de degradación por corrosión localizada, lo que confirma que la película de óxido de cromo generada protege al material frente a este tipo de corrosión (figs. 9b-d).

Un análisis más detallado y a mayores amplificaciones (Figs. $9 \mathrm{~d}$ y f) muestra que los aceros tratados químicamente muestran picaduras de un orden de magnitud menor (10-20 micrómetros) que además parecen encontrarse recubiertos por una fina película de óxido, que induciría a pensar en una repasivación de la picadura. Además, se puede observar, que para el acero tratado por tiempos de 60 minutos, los límites de grano se encuentran revelados, indicio de un proceso de corrosión intergranular, probablemente inducido durante el tratamiento de coloración previo al ensayo de corrosión.

En lo referente al estudio de corrosión del acero AISI 316, la Fig. 10 y Tabla V se muestran los resultados obtenidos a partir del ensayo de polarización anódica a distintas condiciones, sumergido en solución de Hank a $37^{\circ} \mathrm{C}$ durante $1 \mathrm{~h}$.
Fig. 9. MICROFOTOGRAFÍAS ÓPTICAS DEL ACERO AISI 304 DESPUÉS DE SER SOMETIDO AL ENSAYO DE POLARIZACIÓN ANÓDICA EN SOLUCIÓN DE HANK A $37^{\circ} \mathrm{C}$ DURANTE $1 \mathrm{H}$ : A) SIN TRATAMIENTO; TRATAMIENTO DE COLORACIÓN QUÍMICA, B) 40 MIN, C) 50 MIN, D) 60 MIN. E) Y F) DETALLES.
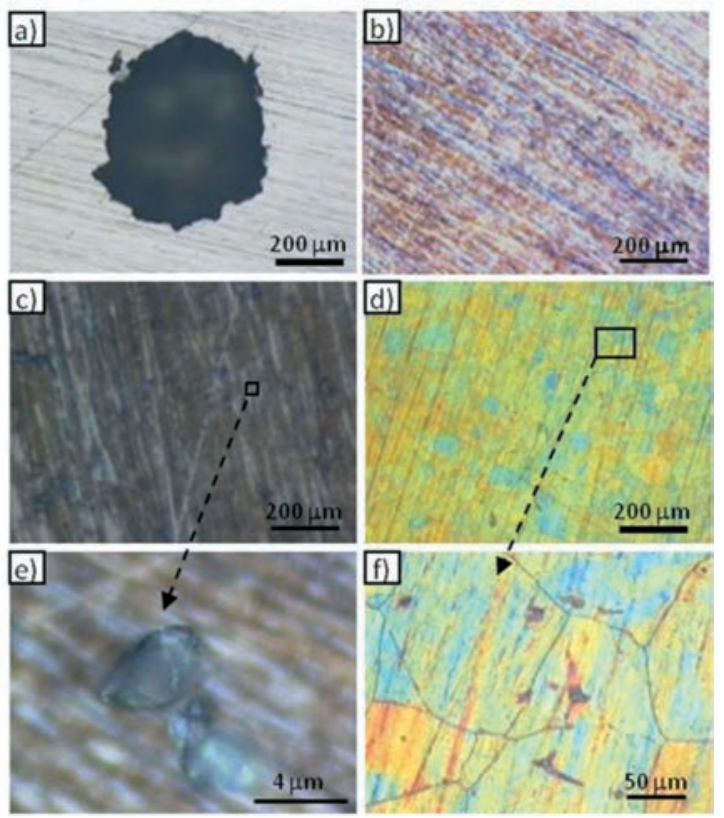

Fig. 10. CURVA DE POLARIZACIÓN ANÓDICA DEL ACERO INOXIDABLE AISI 316L SIN TRATAMIENTO Y TRATADO MEDIANTE COLORACIÓN QUÍMICA, DESPUÉS DE INMERSIÓN EN SOLUCIÓN DE HANK A $37^{\circ} \mathrm{C}$ DURANTE $1 \mathrm{H}$.

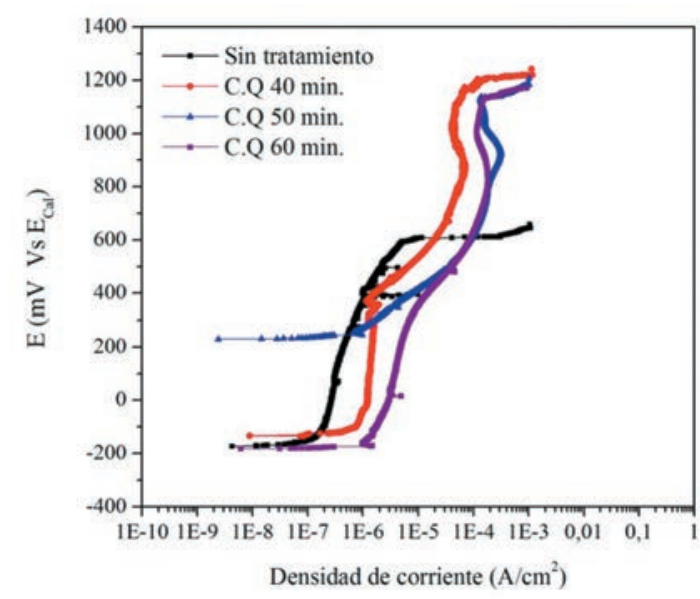


TABLA V

PARÁMETROS CARACTERÍSTICOS DEL ENSAYO DE POLARIZACIÓN ANÓDICA PARA EL ACERO AISI 316L EN DISTINTAS CONDICIONES, SUMERGIDO EN SOLUCIÓN DE HANK A $37^{\circ} \mathrm{C}$ DURANTE 1 HORA

\begin{tabular}{|c|c|c|c|c|c|}
\hline Condición & Ecorr $(\mathrm{mV})$ & Epic $(\mathrm{mV})$ & $\begin{array}{c}\text { Rango de Pasividad } \\
(\mathrm{mV})\end{array}$ & icorr $\left(\mathrm{A} / \mathrm{cm}^{2}\right)$ & ipas $\left(\mathrm{A} / \mathrm{cm}^{2}\right)$ \\
\hline Sin recubrimiento & -170 & 580 & 750 & $8,0 \mathrm{E}-08$ & $6,0 \mathrm{E}-07$ \\
\hline 40 minutos & -115 & 360 & 480 aprox. & $7,7 \mathrm{E}-07$ & $1,4 \mathrm{E}-06$ \\
\hline 50 minutos & -180 & 340 & 520 aprox. & $1,4 \mathrm{E}-06$ & $3,8 \mathrm{E}-06$ \\
\hline 60 minutos & 230 & - & - & $1,0 \mathrm{E}-06$ & - \\
\hline
\end{tabular}

Se observa que para el acero sin recubrimiento, la zona de pasividad del material se extendió por alrededor de $750 \mathrm{mV}$, aproximadamente 3 veces más amplia que para el acero AISI 304, hecho asociado a la presencia de molibdeno que estabiliza la película pasiva e incrementa su resistencia a la corrosión [13].

Con relación a los aceros tratados mediante coloración química, durante tiempos de 40 y 50 minutos, estos presentaron un comportamiento distinto al tratado durante 60 minutos. De este modo, para los primeros se observó una región de pasividad, indicio de la formación de una película protectora superficial que alcanzó un valor cercano a $480 \mathrm{mV}$. Por encima de dicho valor, pareció existir un proceso de corrosión de baja intensidad que tendió a desestabilizar la pasividad de la capa. Sin embargo, no podría considerarse como un fenómeno de corrosión por picadura estable, ya que no existió un aumento drástico de la corriente para un mismo valor de potencial. Por el contrario, el incremento de corriente es ligeramente progresivo a medida que el potencial toma valores mayores. En cuanto a las densidades de corriente de corrosión y pasivación (ipas) se observó que, aunque sus valores se vieron incrementados por los tratamientos de coloración, dicho incremento no parece suponer un cambio significativo en la resistencia a la corrosión del material.

Por otro lado, con relación al acero tratado por tiempos de 60 minutos se pudo observar un comportamiento similar al presentado por el acero AISI 304 tratado durante el mismo tiempo. En este sentido, la curva de polarización anódica no mostró una región definida de pasividad, por lo que induce a pensar en que el material se encuentra desprotegido o sensibilizado de forma previa al ensayo de corrosión a causa del tratamiento de coloración.
Con objeto de complementar el análisis de la resistencia a la corrosión, en la Fig. 11 se muestran el aspecto superficial que presentan los distintos aceros ensayados electroquímicamente durante $1 \mathrm{~h}$ en la solución de Hank. De nuevo se puede observar cómo se pasa de fenómeno de corrosión por picadura bien definido para el acero sin tratar, a una superficie virtualmente libre de picaduras (aunque pueden existir picaduras metaestables) para las muestras con tratamientos químicos de 40 y 50 minutos de duración. Esta observación corrobora lo mostrado por las curvas de polarización anódica, donde no se presentó un aumento drástico de corriente $y$, por tanto, la corrosión sufrida por estos aceros no podría considerarse como corrosión por picadura estable. Finalmente, para tiempos de 60 minutos la superficie muestra de forma clara indicios de una corrosión intergranular previa producida probablemente durante el tratamiento de coloración.

Fig. 11. MICROGRAFíAS ÓPTICAS DEL ACERO AISI 316 L DESPUÉS DE SER SOMETIDO AL ENSAYO DE POLARIZACIÓN ANÓDICA EN SOLUCIÓN DE HANK A $37^{\circ} \mathrm{C}$ DURANTE $1 \mathrm{H}$ : A) SIN TRATAMIENTO; TRATAMIENTO DE COLORACIÓN QUÍMICA, B) 40 MIN, C) 50 MIN, D) 60 MIN
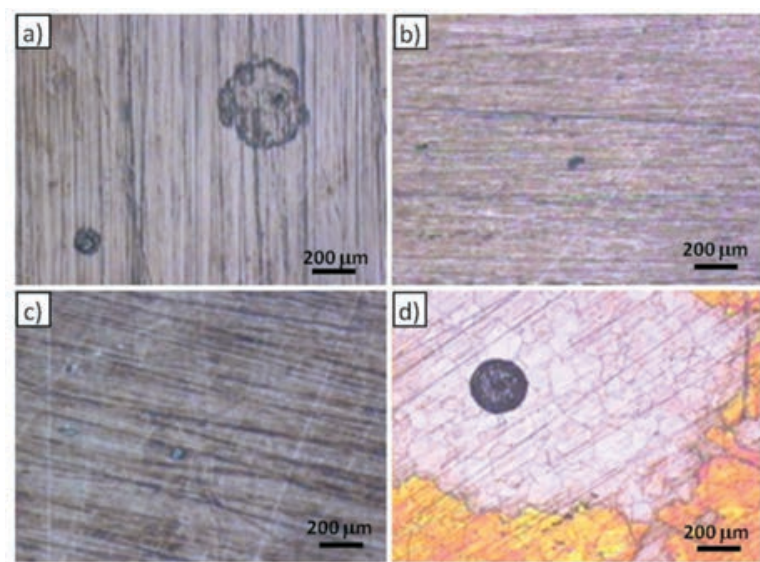


\subsubsection{Coloración química más endurecimiento electroquímico}

Los parámetros electroquímicos de interés determinados a partir del análisis de las curvas de polarización anódica se recogen en la Tabla VI.

En general, el tratamiento de endurecimiento electroquímico tendió a estabilizar la película de óxido de cromo superficial formada durante la coloración química, incluso para el caso de las muestras coloreadas químicamente a 60 minutos que presentaban previamente corrosión intergranular. De esta forma, se puede apreciar cómo, en la mayoría de los casos, los aceros no presentaron un potencial de picadura definido, dando como resultado una región de pasividad más o menos acentuada, indicio de la presencia de una película superficial de carácter protector. Este hecho probablemente se debió al proceso de sellado de poros de la película de óxido producido durante el endurecimiento electroquímico que da al acero un grado de protección extra y limita la difusión de especies a través de su interior [9].

Por otro lado, se puede apreciar cómo un aumento de la densidad de corriente aplicada de 5,0 a $6,0 \mathrm{~mA} / \mathrm{cm}^{2}$ durante el endurecimiento electroquímico favorece la aparición de una región de pasividad mucho más extensa y definida en la curva de polarización, sugiriendo el aumento del carácter protector de la película de óxido. Este hecho probablemente esté asociado al menor grado de porosidad y mayor espesor de las películas formadas para mayores densidades de corriente que incrementarían el grado de protección del acero. Por otro lado, puede apreciarse en líneas generales cómo para ambas densidades de corriente, los aceros coloreados químicamente durante 60 minutos presentan un carácter pasivo ligeramente inferior a los aceros tratados por tiempos inferiores (regiones de pasividad de $700 \mathrm{mV}$ frente $1000 \mathrm{mV}$ ), hecho posiblemente debido a la sensibilización previa por corrosión intergranular causada en el acero.

Los aumentos de $\mathrm{i}_{\text {corr }}$ e $\mathrm{i}_{\text {pas }}$ por el uso de densidades de corriente mayores durante el endurecimiento electroquímico, no son lo suficientemente relevantes como para que deban ser considerados como factores negativos en la resistencia a la corrosión del recubrimiento.

Finalmente, en la Fig. 12 se muestran las micrografías ópticas del aspecto superficial de los aceros coloreados por 40 y 60 minutos y endurecidos electroquímicamente mediante el uso de densidades de corriente de 5,0 y $6,0 \mathrm{~mA} / \mathrm{cm}^{2}$, después del ensayo de polarización anódica.

TABLA VI

PARÁMETROS CARACTERÍSTICOS DEL ENSAYO DE POLARIZACIÓN ANÓDICA PARA LOS ACEROS AISI 304 Y AISI 316L EN DISTINTAS CONDICIONES, SUMERGIDO EN SOLUCIÓN DE HANK A 370C DURANTE 1 HORA

\begin{tabular}{|c|c|c|c|c|c|c|c|}
\hline 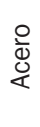 & $\mathrm{t}_{\mathrm{inmersión}}(\mathrm{min})$ & $\begin{array}{l}\text { है } \\
\text { है } \\
\text { है }\end{array}$ & Ecorr $(\mathrm{mV})$ & Epic (mV) & $\begin{array}{l}\text { Rango de pasividad } \\
\text { (mV) Aprox }\end{array}$ & $\operatorname{icorr}\left(\mathrm{A} / \mathrm{cm}^{2}\right)$ & ipas $\left(\mathrm{A} / \mathrm{cm}^{2}\right)$ \\
\hline \multirow{6}{*}{$\begin{array}{l}\frac{J}{\infty} \\
\frac{\bar{m}}{\alpha}\end{array}$} & 40 & \multirow{3}{*}{5,0} & -180 & ND. & 350 & 2,1 E-08 & 8,6 E-07 \\
\hline & 50 & & -540 & 430 & 970 & $1,5 \mathrm{E}-07$ & 1,1 E-05 \\
\hline & 60 & & -130 & ND. & 350 & 6,3 E-08 & 3,6 E-06 \\
\hline & 40 & \multirow{3}{*}{6,0} & -520 & ND. & 1000 & 1,1 E-07 & 1,8 E-05 \\
\hline & 50 & & -520 & ND. & 1000. & 1,2 E-07 & 1,6 E-05 \\
\hline & 60 & & -210 & ND. & 700. & 2,7 E-07 & 6,5 E-06 \\
\hline \multirow{6}{*}{$\begin{array}{l}\overrightarrow{0} \\
\stackrel{-}{m} \\
\frac{\omega}{\sigma}\end{array}$} & 40 & & -140 & ND & 500. & 2,3 E-07 & 3,2 E-06 \\
\hline & 50 & & -250 & ND & 600 & 3,2 E-07 & 6,5 E-06 \\
\hline & 60 & 5,0 & -150 & ND & 350 & 2,5 E-07 & 2,2 E-06 \\
\hline & 40 & \multirow{3}{*}{6,0} & -130 & ND & 550 & 8,9 E-07 & 4,2 E-06 \\
\hline & 50 & & -400 & ND & 800 & 5,2 E-06 & 8,2 E-06 \\
\hline & 60 & & -50 & ND & 500 & 1,3 E-06 & 4,8 E-06 \\
\hline
\end{tabular}

*ND: No definido 
Fig. 12. MICROGRAFÍAS ÓPTICAS DE LOS ACEROS AISI 304 Y AISI $316 \mathrm{~L}$ COLOREADOS QUÍMICAMENTE (CQ) Y ENDURECIDOS ELECTROQUÍMICAMENTE (EE), DESPUÉS DE SER SOMETIDO AL ENSAYO DE POLARIZACIÓN ANÓDICA EN SOLUCIÓN DE HANK A $37^{\circ} \mathrm{C}$ DURANTE $1 \mathrm{H}$.

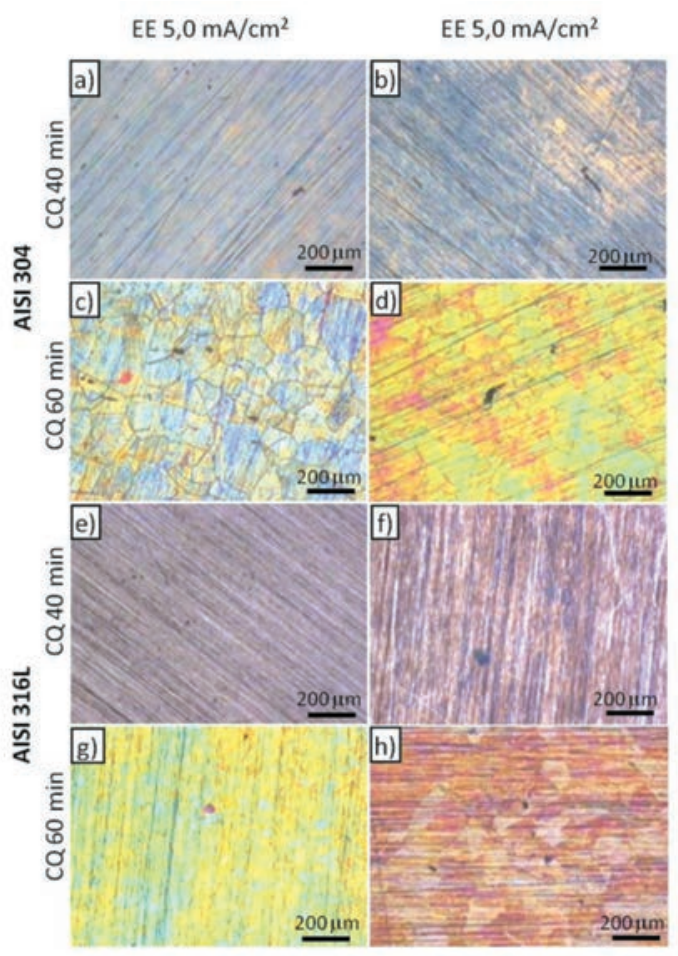

Puede apreciarse que los aceros colorados químicamente a 40 minutos y posteriormente endurecidos presentaron una superficie virtualmente libre de corrosión, aunque no se descarta la presencia de picaduras de pequeño tamaño de carácter metaestable y repasivadas. Por otro lado, con relación a los aceros coloreados durante 60 minutos de inmersión todavía es posible observar la corrosión intergranular generada durante el proceso de coloración química; sin embargo, el crecimiento de la película de óxido durante la etapa de endurecimiento electroquímico parece cubrir parcialmente la superficie del acero sensibilizado dando protección frente al medio agresivo. Este hecho es más notorio cuanto mayor es la densidad de corriente utilizada en el tratamiento

\section{CONCLUSIONES}

El tratamiento de coloración química sobre los aceros inoxidables AISI 304 y AISI 316 L permitió obtener una coloración homogénea y reproducible, de tal forma que se observó la existencia de una relación entre la coloración y el espesor de la película de óxido formada.
El espesor de las películas de óxido de cromo y las coloraciones obtenidas mediante coloración química y posterior tratamiento de endurecimiento electroquímico dependieron tanto del tiempo de inmersión en la etapa química como de la densidad de corriente aplicada a la muestra durante su proceso de endurecimiento.

Las coloraciones obtenidas químicamente sobre los aceros inoxidables AISI 304 y AISI 316L modificaron el mecanismo de corrosión con relación a los aceros base. De este modo, se pasó del convencional ataque localizado por picadura, a un fenómeno de corrosión de tipo generalizado que sucedió a elevados potenciales, gracias a que la superficie del material presentó una mejora sustancial de sus características pasivas.

El tratamiento de endurecimiento electroquímico mejoró sustancialmente las características pasivas de la película de óxido formada, hecho atribuido a la reducción de porosidad de la película formada por coloración química. Un incremento de la densidad de corriente aplicada mejoró más dichas propiedades, posiblemente asociado al aumento del espesor de las películas formadas.

La presencia de molibdeno en la composición del acero inoxidable AISI 316L permitió la obtención de una mayor gama de coloraciones, y estabilizó la película de óxido de cromo, limitando su crecimiento con relación al acero AISI 304, algo que se vio reflejado en los menores espesores de película obtenidos. Por otro lado, dicha estabilización repercutió en la resistencia a la corrosión del acero, observándose un incremento del rango de pasividad.

\section{AGRADECIMIENTOS}

Los autores agradecen al Grupo de Investigación en Desarrollo y Tecnología de Nuevos Materiales - GIMAT y a la Vicerrectoría de Investigación y Extensión de la Universidad Industrial de Santander (Colombia) por el apoyo financiero para el desarrollo de esta investigación a través del macro-proyecto: Desarrollo de Materiales y Recubrimientos de Interés Tecnológico (Código 5450), y al Grupo de Investigación en Corrosión- GIC, de la misma Universidad, por el apoyo técnico. 


\section{REFERENCIAS}

[1] M. Sumita, T. Hanawa, S.H. Teoh. "Development of nitrogen-containing nickel-free austenitic stainless steel for metallic biomaterials- review", Mater. Sci. and Engineer C, Vol. 24, No. 6-8, pp. 753-760, dic. 2004.

[2] M. B. Nasab, M. R. Hassan, "Metallic biomaterials of knee and hip - A review", Trends Biomater.Artif.Organs, Vol. 24 No. 1, pp. 69-82, 2010.

[3] M. Navarro, A. Michiardi, O. Castaño, J. A. Planell. "Biomaterials in orthopedics" J. R Soc. Interface, Vol.5 No. 27, pp. $1137-1158$ oct. 2008.

[4] M. Sumita, Y. Ikada, T. Tateishi (Eds.), "Metallic Biomaterials-Fundamentals and Applications", ICP, Tokyo, 2000 pp. 629.

[5] K. Ogura, W. Lou, M. Nakayama, "Coloration of stainless steel at room temperature by triangular current scan method" J. ElectrochimActa. Vol 41, No. 18, pp 2849-2853, ene.1996.

[6] A., Shahryari, S. Omanovic, J. A. Szpunar "Electrochemical formation of highly pitting resistant passive films on a biomedical grade 316LVM stainless steel surface" Materials Science and Engineering C 28, No. 1, pp. 94-106, ene. 2008.

[7] J.H. Wang, J.G. Duh, H.C. Shih, "Corrosion characteristics of coloured films on stainless steels INCO and a.C process" Surf. Coat. Technol. Vol. 78 No. 1-3, pp 248254, ene. 1996.
[8] R. Conrrado, N. Bocchi, R. C. Rocha-Filho, S. R. Biaggio. "Corrosion resistance of colored films grown on stainless steel by the alternating potential pulse method" ElectrochimicaActa Vol. 48, No. 17 pp. 2417-2428, jul. 2003.

[9] E. Kikuti, N. Bocchi, J.L. Pastol, M.G. Ferreira, M.F. Montemor, M. da Cunha Belo, A.M. Simoes. "Composition and structure of coloured oxide films on stainless steel formed by triangular current scan and cathodic hardening treatment" Corrosion Science, Vol. 49, No. 5, pp. 2303-2314, may 2007.

[10] Z.L. Li, H.Y. Zheng, K.M. Teh, Y.C. Liu, G.C. Lim, H.L. Seng, N.L. Yakovlev. "Analysis of oxide formation induced by UV laser coloration of stainless steel" App. Suf. Sci. Vol. 256, No. 5, pp. 1582-1588. Dic. 2009.

[11] A. J. Antonczak, D. Kocon, M. Nowak, P. Kozioł, K.M. Abramski, "Laser-induced colour marking-Sensitivity scaling for a stainless steel” App. Suf. Sci. Vol. 264, No. 1, pp. 229-236. Ene. 2013.

[12] A. Lehmuskero, V. Kontturi, J. Hiltunen, M. Kuittinen, "Modeling of laser colored stainless steel surfaces by color pixels", Appl. Phys. B 98 pp. 497-500, 2009.

[13] A. Pardo, M.C. Merino, A. E. Coy, F. Viejo, R. Arrabal, E. Matykina. "Pitting corrosion behaviour of austenitic stainless steels - combining effects of $\mathrm{Mn}$ and Mo additions" Corr. Sci, Vol. 50, No. 6 1796-1806 jun. 2008. 